\title{
Catarata infantil: importância do diagnóstico e tratamento precoces
}

\author{
Infantilecataract: the importance of early treatmentand diagnosis
}

\author{
Rafael Vidal Mérula ${ }^{1}$ \\ Luciene Chaves Fernandes ${ }^{2}$
}

Trabalho realizado no Serviço de Visão Subnormal do Hospital São Geraldo - Hospital das Clínicas da Universidade Federal de Minas Gerais (UFMG).

${ }^{1}$ Médico residente do terceiro ano de Oftalmologia do Hospital São Geraldo, Hospital das Clínicas da Faculdade de Medicina da Universidade Federal de Minas Gerais (UFMG). Belo Horizonte (MG).

${ }^{2}$ Doutora em Oftalmologia pela Faculdade de Medicina da UFMG. Belo Horizonte (MG).

Endereço para correspondência: Rafael Vidal Mérula Rua Alameda Álvaro Celso, 250 - apto. 206 - Belo Horizonte (MG) CEP 30150-260

E-mail: rafaelmerula@hotmail.com

Recebido para publicação em 24.10.2003

Versão revisada recebida em 08.09.2004

Aprovação em 02.02.2005

\section{RESUMO}

Objetivos: Estabelecer freqüência da catarata infantil no Serviço de Visão Subnormal-Hospital São Geraldo (HCUFMG), analisar dados da anamnese, exame oftalmológico e prescrição óptica nesses pacientes. Métodos: Estudo observacional, descritivo e retrospectivo. Foram analisados prontuários de catarata infantil do Serviço de Visão Subnormal, de janeiro/1992 a dezembro/2002 referentes à idade, sexo, cor, idade da primeira observação da leucocoria e quem a observou, idades do diagnóstico e realização da facectomia, história familiar de catarata, implante de lente intra-ocular, acuidade visual (AV) e prescrição óptica. Resultados: Foram estudados 44 prontuários. Faixa etária de 0 a 15 anos, sendo $19(43,0 \%)$ de 0 a 3 anos, 14 $(32,0 \%)$ de 4 a 10 anos, $11(25,0 \%)$ de 11 a 15 anos. Vinte e oito $(63,6 \%)$ do sexo feminino. Dezenove $(43,2 \%)$ leucodérmicos. Primeira observação da leucocoria foi feita pela mãe em 17 pacientes $(38,6 \%)$. Observação de leucocoria ocorreu em $45,5 \%$ dos pacientes nos primeiros 2 meses de vida. Facectomia foi realizada em $43,2 \%$ dos pacientes acima de 1 ano. Prescrição de óculos foi realizada em $61,4 \%$, sendo em $9,1 \%$ abaixo de 12 meses de idade. Lente intra-ocular foi implantada em $13,6 \%$ dos pacientes. Quatro $(9,1 \%)$ tinham história familiar de catarata congênita. Em 20,4\% a AV foi menor que 20/400. Glaucoma secundário ocorreu em $18,2 \%$ dos pacientes. Prescrição de auxílios ópticos incluiu 2 óculos e 1 telescópio. Conclusões: Mais informações sobre catarata congênita devem ser dadas aos familiares e pediatras, facectomia deve ser realizada precocemente, correção óptica e tratamento da ambliopia devem ser instituídas prontamente.

Descritores: Catarata/congênita; Catarata/diagnóstico; Catarata/epidemiologia; Baixa visão

\section{INTRODUÇÃO}

Define-se catarata infantil a opacificação do cristalino que reduz a visão em indivíduos de 0 a 15 anos $^{(1)}$.

Apresenta incidência de 1/2000 nascidos vivos e 10 novos casos/milhão população/ano. Estima-se que ela seja responsável por 10-38,8\% de toda a cegueira prevenível e tratável na infância (AV corrigida inferior a 20/400 ou 0,05 no melhor olho em indivíduos menores de 16 anos, segundo a Organização Mundial de Saúde) em crianças de todo o mundo ${ }^{(2)}$. A prevalência em crianças é de 1 a 4/10.000 nos países em desenvolvimento e aproximadamente 0,1 a $0,4 / 10.000$ nos países desenvolvidos ${ }^{(3)}$. Considera-se que 40 a $50 \%$ dos portadores de catarata infantil apresentam baixa visão ${ }^{(1)}$.

Pode ocorrer como alteração isolada ou como parte de um quadro ocular e/ou sistêmico. Pode ser idiopática - cerca de metade das cataratas infantis são idiopáticas ${ }^{(3)}$ ou estar relacionada a diversos fatores como: doenças sistêmicas de origem genética (Trissomia 21, Síndrome de Bardet-Biedl), 
metabólica (galactosemia, hipoglicemia, hipocalcemia), infecções intra-uterinas (rubéola, toxoplasmose), induzida por drogas (corticosteróides, clorpromazina), traumatismo, prematuridade, outras doenças oculares (microftalmia, aniridia, tumor intra-ocular, retinopatia da prematuridade).

$\mathrm{O}$ sucesso visual na catarata infantil relaciona-se com a precocidade do diagnóstico e do correto tratamento instituí$\mathrm{do}^{(3)}$. Os avanços nas técnicas cirúrgicas têm reduzido os índices de complicações. Contudo, ações mais efetivas devem ser instituídas buscando prevenção, diagnóstico e tratamento mais precoces, adequada correção óptica, tratamento da ambliopia associados à intervenção precoce, para uma melhor qualidade de vida das crianças.

\section{OBJETIVO}

Estabelecer freqüência da catarata infantil no Serviço de Visão Subnormal do Hospital São Geraldo (HCUFMG) e analisar dados da anamnese, exame oftalmológico e prescrição óptica nesses pacientes.

\section{MÉTODOS}

Foram analisados retrospectivamente os prontuários de todos os pacientes com diagnóstico de catarata congênita matriculados no Serviço de Visão Subnormal do HSG entre janeiro de 1992 e dezembro de 2002 e selecionados aqueles com idade entre zero e 15 anos (catarata infantil).

Como rotina do Serviço, os pacientes já submetidos a exame oftalmológico completo, eram encaminhados para avaliação especializada em visão subnormal que constava de anamnese, medida da acuidade visual para longe e perto através das tabelas LH (Lea Hyvarinen), para os iletrados e ETDRS (Early Treatment Diabetic Retinopathy Study), para os letrados e os Cartões de Teller nas crianças que não respondiam aos outros testes. Corrigiu-se, então, a ametropia e realizou-se a prescrição óptica e não óptica necessária. Naqueles pacientes sem correção da ametropia, esta foi feita no Serviço. O paciente foi, então, incluído em programa de intervenção precoce, potencialização da visão residual e utilização do auxílio óptico ou eletrônico a serem prescritos. Para o cálculo da magnificação, é rotina do Serviço de Visão Subnormal do Hospital São Geraldo utilizar a regra de Kestenbaum (ampliação $=1 / \mathrm{AV}$ ) como referência inicial, e isto é feito somente para crianças em fase pré-escolar e escolar que irão utilizar auxílios ópticos, tendo como objetivo alcançar 20/50.

Foram levantados dados referentes à idade, sexo, cor, idade da primeira observação da leucocoria e por quem ela foi observada, idades do diagnóstico da catarata e da realização da facectomia, o implante de lente intra-ocular, história familiar de catarata, idade da prescrição óptica da ametropia, acuidade visual e prescrição de auxílios ópticos.

Todos os dados foram anotados em um protocolo específico e então analisados.
A faixa etária foi classificada como: grupo I - crianças de 0 a 3 anos, grupo II - de 4 a 10 anos, e grupo III - de 11 a 15 anos.

A distribuição da acuidade visual corrigida no melhor olho seguiu os parâmetros de comprometimento visual da Classificação Internacional de doenças - Décima revisão.

- Grupo 1:20/30 a 20/60

- Grupo 2: <20/60 a 20/160

- Grupo 3:20/200 a 20/400

- Grupo 4: 20/500 a 20/1000

- Grupo 5: 20/1250 a percepção luminosa

- Grupo 6: sem percepção de luz

Os valores de AV foram transformados em logMAR, e a partir daí, foram calculados média, mediana e desvio padrão.

Nas crianças de 0-3 anos, o valor de normalidade da AV corrigida no melhor olho baseou-se nas normas contidas no manual dos cartões de Teller ${ }^{(4)}$ :

Crianças 2 meses: 20/360 a 20/1400

Crianças 4 meses: 20/180 a 20/670

Crianças 6 meses: 20/130 a 20/540

Crianças 9-12 meses: 20/94 a 20/380

Crianças 18-24 meses: 20/47 a 20/190

Crianças 30-36 meses: 20/16 a 20/63

Com referência aos auxílios em visão subnormal, considerou-se auxílios para perto os óculos com adição especial, as lupas de mão (LM), as lupas de apoio (LA) e o circuito fechado de TV (CCTV) e, para longe, o telescópio (TS).

\section{RESULTADOS}

Foram analisados $65(4,9 \%)$ prontuários com diagnóstico de catarata congênita do Serviço de Visão Subnormal do Hospital São Geraldo, de um total de 1.332 prontuários e selecionados $44(3,3 \%)$ - freqüência de catarata infantil no ambulatório de Visão Subnormal (Tabela 1). Vinte e um foram excluídos $(32,3 \%)$ por apresentarem idade superior a 15 anos $(81 \%)$, dados incompletos $(14,3 \%)$ e catarata pós-uveíte $(4,7 \%)$. A faixa etária, variou de 0 a 15 anos, sendo 19 pacientes $(43,0 \%)$ de 0 a 3 anos, $14(32,0 \%)$ de 4 a 10 anos, $11(25,0 \%)$ de 11 a 15 anos (Gráfico 1). Dezesseis $(36,4 \%)$ eram do sexo masculino e $28(63,6 \%)$ do sexo feminino. Dezenove $(43,2 \%)$ eram leucodérmicos, $8(18,2 \%)$ feodérmicos, $2(4,5 \%)$ melanodérmicos e 15 $(34,1 \%)$ desconhecida. A primeira observação de leucocoria ocorreu em 20 pacientes $(45,5 \%)$ com idade menor ou igual a 2 meses, em $4(9,1 \%)$ de 3 a 6 meses, em $1(2,3 \%)$ de 7 a 11 meses, em $3(6,8 \%)$ de 12 a 48 meses, em $1(2,3 \%)$ acima de 48 meses, o dado era desconhecido em 15 (34,0\%) (Gráfico 2). A observação da leucocoria foi realizada pela mãe em $17(38,6 \%)$ casos, pelo pai em $1(2,3 \%)$, pela avó em $1(2,3 \%)$, pelo pediatra em 1 $(2,3 \%)$, pela tia em $1(2,3 \%)$ e o dado era desconhecido em 23 pacientes $(52,3 \%)$ (Tabela 2$)$. A facectomia foi realizada em 42 pacientes $(95,5 \%)$, sendo que em $2(4,5 \%)$ na faixa etária menor ou igual que 2 meses, em $17(38,6 \%)$ de 3 a 6 meses, em $4(9,1 \%)$ de 7 a 11 meses, em $17(38,6 \%)$ de 12 a 48 meses, em $2(4,6 \%)$ com idade maior que 48 meses, em $1(2,3 \%)$ paciente o dado era 


\begin{tabular}{|c|c|c|c|c|c|c|c|}
\hline ID & $\begin{array}{l}\text { Idade } \\
(\mathrm{m} / \mathrm{a})\end{array}$ & $\begin{array}{l}\text { Idade da } 1^{\mathrm{a}} \\
\text { observação de } \\
\text { leucocoria }(\mathrm{m})\end{array}$ & $\begin{array}{c}\text { Idade de } \\
\text { realização da } \\
\text { facectomia }(\mathrm{m})\end{array}$ & $\begin{array}{c}\text { Idade da } \\
\text { correção óptica } \\
\text { pós-operatória }(\mathrm{m})\end{array}$ & $\begin{array}{l}\text { Implante } \\
\text { de lente } \\
\text { intra-ocular }\end{array}$ & $\begin{array}{l}\text { Acuidade visual } \\
\text { (Snellen/logMAR) }\end{array}$ & $\begin{array}{l}\text { Auxílios } \\
\text { ópticos }\end{array}$ \\
\hline 1. LAAL & $9 \mathrm{~m}$ & 1 & 2 & $\mathrm{~d}$ & Sim & $20 / 260$ / 1,11 & Não \\
\hline 2. DAS & $11 \mathrm{~m}$ & $d$ & 6 & 11 & Não & $20 / 170 / 0,92$ & Não \\
\hline 3. MEFRS & $12 \mathrm{~m}$ & 5 & 5 & 6 & $d$ & $20 / 710 / 1,50$ & Não \\
\hline 4. ALCR & $13 \mathrm{~m}$ & 1 & 4 & 31 & Não & $20 / 800 / 1,60$ & Não \\
\hline 5. MECF & $14 \mathrm{~m}$ & 5 & 11 & 14 & Não & $20 / 1400 / 1,85$ & Não \\
\hline 6. CGM & $17 \mathrm{~m}$ & 1 & 6 & $d$ & Não & $20 / 710 / 1,50$ & Não \\
\hline 7. SMSS & $18 \mathrm{~m}$ & $d$ & 3 & 18 & Não & $20 / 125 / 0,80$ & Não \\
\hline 8. RMS & $18 \mathrm{~m}$ & $\mathrm{n}$ & $d$ & $d$ & $d$ & $20 / 250 / 1,10$ & Não \\
\hline 9. MBS & $18 \mathrm{~m}$ & 0 & 4 & $d$ & Não & $20 / 940 / 1,70$ & Não \\
\hline 10. PCM & $24 \mathrm{~m}$ & $d$ & 3 & 13 & Não & $20 / 64 / 0,50$ & Não \\
\hline 11. LAS & $24 \mathrm{~m}$ & $\mathrm{n}$ & 12 & $d$ & $d$ & $20 / 1400 / 1,85$ & Não \\
\hline 12. JSMR & $24 \mathrm{~m}$ & 0 & 4 & 6 & Não & $20 / 200 / 1,00$ & Não \\
\hline 13. LCS & $24 \mathrm{~m}$ & 2 & 18 & $d$ & $d$ & $20 / 960 / 1,70$ & Não \\
\hline 14. LMN & $24 \mathrm{~m}$ & 1 & 3 & 20 & Não & $20 / 250 / 1,10$ & Não \\
\hline 15. JAS & $26 \mathrm{~m}$ & $d$ & 24 & 24 & Sim & $20 / 1400 / 1,85$ & Não \\
\hline 16. TCSM & $31 \mathrm{~m}$ & $d$ & 5 & $d$ & Não & $20 / 125 / 0,80$ & Não \\
\hline 17. GRS & $36 \mathrm{~m}$ & $d$ & 36 & $d$ & Não & $20 / 710 / 1,50$ & Não \\
\hline 18. ALCC & $36 \mathrm{~m}$ & 2 & 48 & $d$ & Sim & $20 / 200 / 1,00$ & Não \\
\hline 19. TCL & $41 \mathrm{~m}$ & 0 & Não & Não & $d$ & $20 / 125 / 0,80$ & Não \\
\hline 20. KAM & $44 \mathrm{~m}$ & 24 & 48 & $d$ & Sim & $20 / 100 / 0,70$ & Não \\
\hline 21. ED & $5 \mathrm{a}$ & 12 & 24 & $d$ & Sim & $20 / 64 / 0,50$ & Não \\
\hline 22. SLF & $6 \mathrm{a}$ & 12 & 24 & $d$ & Sim & $20 / 200 / 1,00$ & Não \\
\hline 23. SGD & $6 \mathrm{a}$ & $\mathrm{n}$ & 2 & 24 & Não & $20 / 300 / 1,20$ & Não \\
\hline 24. APD & $7 \mathrm{a}$ & $\mathrm{n}$ & 4 & 24 & Não & $20 / 80 / 0,60$ & Não \\
\hline 25. MPSC & $7 \mathrm{a}$ & $d$ & 6 & 40 & Não & $20 / 200 / 1,00$ & Não \\
\hline 26. RMJ & $7 \mathrm{a}$ & $d$ & 4 & 5 & Não & $20 / 150 / 0,86$ & Não \\
\hline 27. NSP & $7 \mathrm{a}$ & $d$ & 8 & 48 & Não & $20 / 64 / 0,50$ & Não \\
\hline 28. PRD & $8 \mathrm{a}$ & 8 & 24 & $d$ & $d$ & $20 / 100 / 0,70$ & Não \\
\hline 29. DLA & $8 \mathrm{a}$ & $\mathrm{n}$ & 48 & 60 & Não & $20 / 32$ / 0,20 & Não \\
\hline 30. JCHS & $9 \mathrm{a}$ & $d$ & 17 & 30 & $d$ & $20 / 200 / 1,00$ & Não \\
\hline 31. FVSR & $9 a$ & $d$ & 7 & 36 & Não & $20 / 250 / 1,10$ & Não \\
\hline 32. AAC & $9 a$ & 0 & 6 & 48 & Não & $20 / 100 / 0,70$ & Sim (óculos) \\
\hline 33. KRS & $10 a$ & $d$ & 12 & $d$ & Não & $20 / 64 / 0,50$ & Não \\
\hline 34. JLS & $11 \mathrm{a}$ & $\mathrm{n}$ & 3 & $d$ & Não & $20 / 64 / 0,50$ & Não \\
\hline 35. MTBS & $11 \mathrm{a}$ & $\mathrm{n}$ & 5 & 21 & Não & $20 / 160 / 0,90$ & Não \\
\hline 36. GLPS & $11 \mathrm{a}$ & $\mathrm{n}$ & 36 & 36 & Não & $20 / 160 / 0,90$ & Não \\
\hline 37. DAT & $12 \mathrm{a}$ & $d$ & 3 & 14 & $d$ & $20 / 125 / 0,80$ & Não \\
\hline 38. DBS & $13 a$ & $d$ & 12 & 14 & Não & $20 / 160 / 0,90$ & Não \\
\hline 39. LMC & $13 a$ & $d$ & 36 & $d$ & Não & $20 / 400 / 1,30$ & Não \\
\hline 40. CFC & $13 \mathrm{a}$ & 5 & 60 & 48 & Não & $d$ & Não \\
\hline 41. ARF & $14 \mathrm{a}$ & 2 & 11 & 36 & $d$ & $20 / 80 / 0,60$ & Não \\
\hline 42. DB & $14 \mathrm{a}$ & 3 & 24 & 40 & $d$ & $20 / 320 / 1,20$ & Não \\
\hline 43. KKS & $15 \mathrm{a}$ & $\mathrm{n}$ & 24 & $d$ & Não & $20 / 160 / 0,90$ & Não \\
\hline 44. LPA & $15 \mathrm{a}$ & 120 & 144 & 144 & Não & $20 / 250 / 1,10 \varsigma$ & Sim (óculos+TS) \\
\hline
\end{tabular}

desconhecido e $1(2,3 \%)$ paciente não realizou cirurgia (Gráfico 3). O implante de lente intra-ocular (LIO) foi realizado em 6 pacientes $(13,6 \%)$, em $28(63,7 \%)$ não se implantou a LIO, e em $10(22,7 \%)$ o dado era desconhecido. A correção óptica pósfacectomia ocorreu em 4 pacientes $(9,1 \%)$ com idade menor que 12 meses, em $10(22,6 \%)$ com idade de 12 a 24 meses, em
$5(11,4 \%)$ com idade de 25 a 36 meses, em $5(11,4 \%)$ de 37 a 48 meses, em 1 (2,3\%) de 49 a 60 meses de idade, em $1(2,3 \%)$ com idade maior que 60 meses, em $17(38,6 \%)$ o dado era desconhecido e $1(2,3 \%)$ paciente não realizou cirurgia (Gráfico 4). A medida da acuidade visual mostrou, na faixa etária de 0 a 3 anos, 4 pacientes $(9,1 \%)$ com AV menor que 20/60 a 20/160, 5 


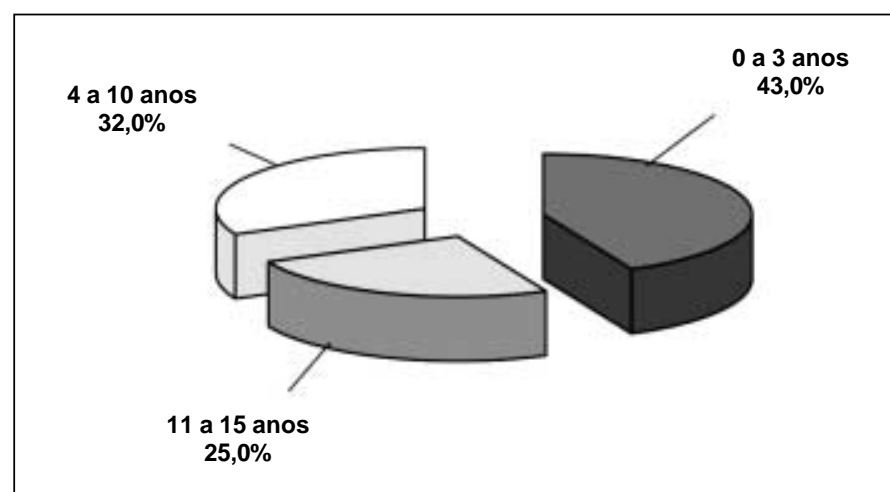

Gráfico 1 - Distribuição por faixa etária dos pacientes com catarata infantil matriculados no Serviço de Visão Subnormal do Hospital São Geraldo (HC-UFMG), entre 1992 - 2002

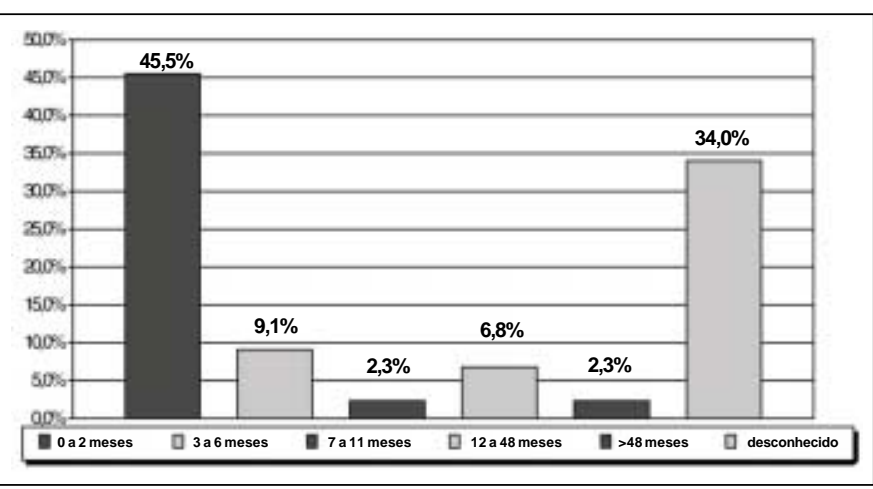

Gráfico 2 - Distribuição por idade da 1a observação da leucocoria dos pacientes com catarata infantil matriculados no Serviço de Visão Subnormal do Hospital São Geraldo (HC-UFMG), entre 1992 - 2002

\begin{tabular}{|c|c|c|}
\hline & $\mathrm{n}$ & $\%$ \\
\hline Mãe & 17 & 38,6 \\
\hline Pai & 1 & 2,3 \\
\hline Avó & 1 & 2,3 \\
\hline Pediatra & 1 & 2,3 \\
\hline Tia & 1 & 2,3 \\
\hline Desconhecido & 23 & 52,3 \\
\hline Total & 44 & 100,0 \\
\hline
\end{tabular}

$(11,4 \%)$ de $20 / 200$ a 20/400, $6(13,6 \%)$ de $20 / 500$ a 20/1000, 3 $(6,8 \%)$ com AV inferior a 20/1000; na faixa etária de 4 a 10 anos, verificou-se 1 paciente $(2,3 \%)$ com AV de $20 / 30$ a 20/60, 8 $(18,1 \%)$ com AV menor que 20/60 a 20/160, $5(11,4 \%)$ de 20/200 a 20/400, 1 (2,3\%) de AV desconhecida; e na faixa etária de 11 a 15 anos, 7 pacientes $(15,9 \%$ ) com AV menor que $20 / 60$ a 20/160, $3(6,8 \%)$ de $20 / 200$ a 20/400, e $1(2,3 \%)$ de AV desconhecida (Tabela 3). A média da AV é 20/150 (0,87 logMAR), a mediana é 20/200 (1,0 logMAR), e o desvio padrão é de $\pm 0,17 \log$ MAR. Em relação à história familiar, verificou-se consangüinidade em 1

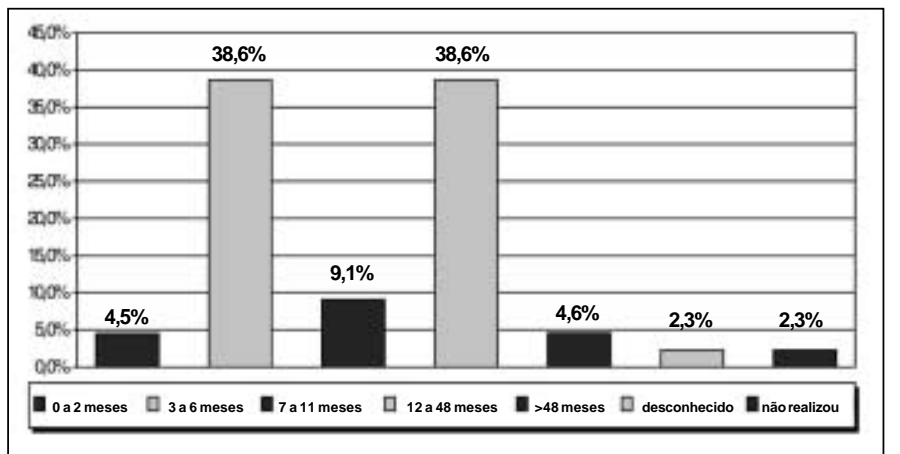

Gráfico 3 - Distribuição por idade da facectomia dos pacientes com catarata infantil matriculados no Serviço de Visão Subnormal do Hospital São Geraldo (HC-UFMG), entre 1992 - 2002

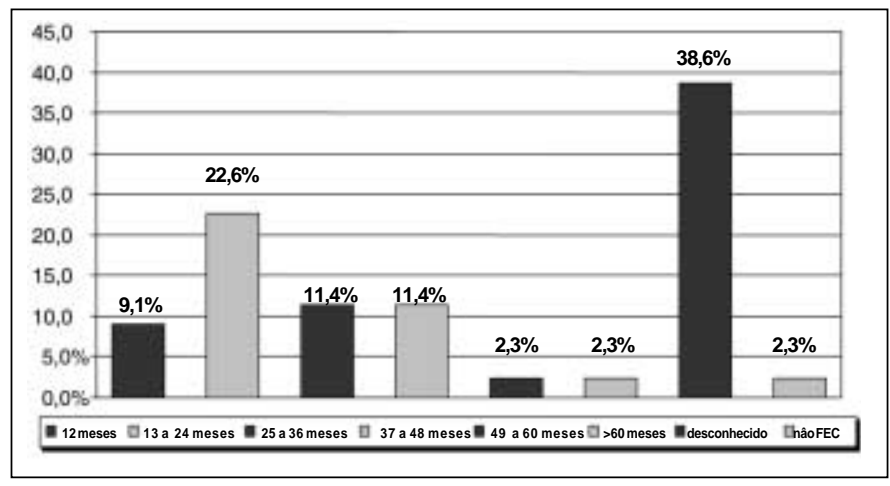

Gráfico 4 - Distribuição por idade da correção óptica pós-facectomia dos pacientes com catarata infantil matriculados no Serviço de Visão Subnormal do Hospital São Geraldo (HC-UFMG), entre 1992 - 2002

\begin{tabular}{|c|c|c|c|c|c|}
\hline \multirow{2}{*}{$\begin{array}{l}\text { Acuidade } \\
\text { visual }\end{array}$} & \multicolumn{3}{|c|}{ Idade (anos) } & \multirow{2}{*}{\multicolumn{2}{|c|}{ Total }} \\
\hline & $\overline{0-3}$ & $4-10$ & $11-15$ & & \\
\hline $20 / 30$ a $20 / 60$ & - & 1 & - & 1 & $(2,3 \%)$ \\
\hline$<20 / 60$ a $20 / 160$ & 4 & 8 & 7 & 19 & $(43,2 \%)$ \\
\hline $20 / 200$ a $20 / 400$ & 5 & 5 & 3 & 13 & $(29,5 \%)$ \\
\hline $20 / 500$ a $20 / 1000$ & 6 & - & - & 6 & $(13,6 \%)$ \\
\hline 20/1250 a PL & 3 & - & - & 3 & $(6,8 \%)$ \\
\hline Desconhecida & - & 1 & 1 & 2 & $(4,6 \%)$ \\
\hline Total & $18(40,9 \%)$ & $15(34,1 \%)$ & $11(25 \%)$ & 44 & $(100,0 \%)$ \\
\hline
\end{tabular}

paciente $(2,3 \%)$, catarata congênita em $4(9,1 \%), 1(2,3 \%)$ com hipotireoidismo, $2(4,5 \%)$ com rubéola gestacional, $1(2,3 \%)$ com estrabismo, e $35(79,5 \%)$ o dado era desconhecido (Tabela 4). Ao analisar a história de doença ocular associada pósfacectomia, constatou-se 8 pacientes $(18,2 \%)$ com glaucoma, $3(6,8 \%)$ com estrabismo, 5 (11,4\%) com nistagmo, $2(4,5 \%)$ com nistagmo e estrabismo e em $26(59,1 \%)$ o dado era desconhecido (Tabela 5). Verificou-se o uso de auxílios ópticos em 2 pacientes: em 1 deles $(2,3 \%)$ utilizaram-se óculos com adição especial e no outro $(2,3 \%)$ óculos e telescópio. 


\begin{tabular}{|lcc|}
\hline \multicolumn{3}{|c|}{$\begin{array}{c}\text { Tabela 4. História familiar dos pacientes com catarata infantil, } \\
\text { matriculados no Serviço de Visão Subnormal do Hospital São } \\
\text { Geraldo (HC-UFMG), entre }\end{array}$} \\
& $\mathbf{1 9 9 2}$ - 2002 & \\
Pais consangüíneos & $\mathbf{n}$ & $\%$ \\
Catarata congênita & 4 & 2,3 \\
Hipotireoidismo & 1 & 9,1 \\
Rubéola gestacional & 2 & 2,3 \\
Sífilis & 1 & 4,5 \\
Desconhecido & 35 & 2,3 \\
Total & 44 & 79,5 \\
\hline
\end{tabular}

\begin{tabular}{lcr|}
$\begin{array}{l}\text { Tabela 5. Doença ocular associada nos pacientes com catarata } \\
\text { infantil, matriculados no Serviço de Visão Subnormal do Hospital } \\
\text { São Geraldo (HC-UFMG), entre }\end{array}$ \\
& $\mathbf{1 9 9 2}$ - 2002 & \\
& $\mathbf{n}$ & $\%$ \\
Glaucoma & 8 & 18,2 \\
Estrabismo & 3 & 6,8 \\
Nistagmo & 5 & 11,4 \\
Nistagmo + Estrabismo & 2 & 4,5 \\
Desconhecido & 26 & 59,1 \\
Total & 44 & 100,0 \\
\hline
\end{tabular}

\section{DISCUSSÃO}

A catarata infantil destaca-se como importante causa de baixa visão na infância. Estudo realizado pela OMS em escolas de cegos no mundo, mostra a catarata infantil como responsável por 8 a $23 \%$ de toda a cegueira na infância: América Latina $8 \%$, África $10 \%$, Ásia $12 \%$ e Europa $23 \%{ }^{(1)}$.

Estudos realizados em Serviços de Visão Subnormal no Brasil mostram a freqüência de catarata congênita variando de 5,8 a $13,0 \%{ }^{(5-10)}$. Neste estudo, a catarata infantil ocorreu em $3,3 \%$ dos casos, possivelmente pelo limite da faixa etária não referido nos trabalhos pesquisados. Segundo trabalho realizado em nosso Serviço de Visão Subnormal, encontrou-se uma frequiência de 5,8\% para catarata congênita ${ }^{(8)}$.

A conduta na catarata congênita não é tarefa simples, uma vez que diversos fatores estão envolvidos como idade do paciente, olho contralateral, tamanho, intensidade e localização da opacidade; doenças sistêmicas e/ou oculares associadas, cirurgia, correção da ametropia, tratamento da ambliopia e a estimulação visual.

Sabemos que no recém-nascido, o sistema nervoso central e, conseqüentemente o sistema visual, é pouco desenvolvido. Todos nós nascemos com baixa visão ${ }^{(10)}$. A partir do nascimento há evolução orgânica e funcional do sistema visual se utilizado adequadamente. Qualquer fator que impeça o desenvolvimento normal da visão pode causar alterações anatômicas e funcionais do sistema visual, levando à baixa visão.

A ambliopia de privação (ex-anopsia) decorrente de catarata congênita é uma forma grave de ambliopia, em que se verifica alteração estrutural e funcional profunda como atrofia das camadas celulares do corpo geniculado lateral relacionada com o olho lesado, bem como redução das respostas eletroencefalográficas occipitais ${ }^{(5)}$.

Assim, o diagnóstico precoce da catarata congênita, bem como a realização da facectomia e correção da ametropia em tempo hábil determinam a resposta visual do indivíduo. Todos os recém-nascidos deveriam ter o reflexo vermelho do fundo do olho obrigatoriamente pesquisado através do oftalmoscópio direto pelo pediatra e repetido dentro de três meses de vida ${ }^{(11)}$. Fato este que já é realidade em alguns estados brasileiros.

Dado importante a destacar neste estudo foi a primeira observação da leucocoria que ocorreu em 20 pacientes $(45,5 \%)$ nos primeiros 2 meses de idade. Entretanto, somente em 2 pacientes $(4,5 \%)$ a facectomia foi realizada até 2 meses. Acreditamos que tal fato espelha a desinformação e a dificuldade de acesso da nossa população a serviços especializados. A realização da facectomia nas primeiras 7 semanas permitirá uma melhor resposta visual ${ }^{(12)}$. O prognóstico pode ser pior quando se posterga a facectomia na catarata congênita unilateral $^{(11-13)}$.

A primeira observação da leucocoria foi feita em $38,6 \%$ dos casos pela mãe o que se justifica pelo maior convívio com a criança. Se houvesse mais orientação e informação para a gestante haveria diagnóstico mais precoce da catarata congênita, uma vez que estas encaminhariam seus filhos para exame oftalmológico mais cedo. Além disso, não devemos esquecer que o neonatologista e o pediatra também desempenham papel fundamental no reconhecimento da leucocoria. Em relação à história familiar observou-se que em 9,1\% dos casos houve a presença da catarata congênita reafirmando fator hereditário, também observado por outro trabalho ${ }^{(11)}$. A ocorrência de glaucoma secundário em 18,2\% dos casos mostra a importância de acompanhamentos clínico destas crianças. A realização da facectomia, em crianças com catarata congênita, está relacionada não somente com a melhora da AV, mas também com a diminuição ou eliminação de sintomas clínicos como: estrabismo e nistag$\mathrm{mo}^{(15)}$. A cirurgia deve ser realizada o mais precocemente possível, uma vez que o prognóstico fica prejudicado após doze semanas de vida, quando se instala nistagmo por privação ${ }^{(2)}$. Neste estudo, o nistagmo foi observado em 7 pacientes $(15,9 \%)$, sendo que em 2 casos havia estrabismo simultaneamente. Em estudo realizado em 2001 evidenciou-se uma redução de 76,2\% do nistagmo 6 meses após facectomia ${ }^{(15)}$.

Estima-se que a catarata infantil seja responsável por 10-20\% de toda a cegueira na infância. Nosso estudo encontrou que $20,4 \%$ dos pacientes apresentam AV menor que 20/400 com média de 20/150 (0,87 logMAR). A acuidade visual variou de 20/32 a menor que 20/1400: 0-3 anos, de 20/60 a PL; 4-10 anos, de 20/30 a 20/400; 11 a 15 anos, de 20/60 a 20/400 (Tabela 1). Em estudo realizado em 1995, observou-se uma visão menor que 20/100 em $75 \%$ de crianças portadoras de catarata congênita, com até 84 meses de idade, submetidas a tratamento cirúrgico ${ }^{(16)}$.

$\mathrm{O}$ prognóstico da catarata congênita nos casos não complicados é bom se a correção for feita prontamente ${ }^{(9)}$. A cirurgia e a correção óptica precoces, no período sensível do 
desenvolvimento visual binocular, devem ser instituídas, especialmente na presença de opacidades densas ${ }^{(17)}$. Por conseguinte, não se deve esperar a idade escolar para fazer a correção da ametropia ${ }^{(9)}$. Estaríamos transformando uma ambliopia de privação em uma ambliopia refracional ${ }^{(9)}$. Em nosso estudo, houve prescrição óptica em $61,4 \%$ dos pacientes e implante de lente intra-ocular em 13,6\% deles. Contudo, a prescrição óptica de pacientes com menos de 12 meses de idade somente ocorreu em 9,1\% dos casos. O implante primário da LIO nos 2 primeiros anos de vida é ainda tema controverso em função da deficiente previsibilidade do cálculo biométrico em relação ao crescimento do olho, biocompatibilidade e pelas possíveis complicações. Por ocasião deste estudo, não era rotina do hospital o implante de lente intra-ocular abaixo de 2 anos. O momento da realização da cirurgia da catarata continua ser mais importante que o método de correção óptica da afacia, principalmente em cataratas congênitas monolaterais ${ }^{(18)}$.

A prescrição de auxílios ópticos especiais depende de fatores como acuidade visual, idade do paciente, necessidades definidas, retardo em diagnosticar e tratar a catarata, bem como, a capacidade de resposta ao auxílio, fatores estes, que justificaram o baixo número de auxílios prescritos (4,6\%). Além disto, na criança utiliza-se muito da magnificação pela aproximação, buscando melhor utilização da visão residual ${ }^{(19)}$. O telescópio é o único auxilio óptico para longe. A realização do estudo em indivíduos na idade escolar, necessitando leitura na lousa, justifica a sua prescrição. Mas, sabemos das dificuldades no uso do telescópio e de suas limitações ${ }^{(20)}$.

Os avanços das técnicas cirúrgicas têm reduzido em muito os índices de complicações no pós-operatório. Salientamos que $6(13,6 \%)$ pacientes deste trabalho obtiveram resultados visuais bons para catarata infantil quando se diagnosticou precocemente e se instituiu tratamento imediato (Tabela 1). Contudo, infelizmente os resultados visuais continuam pobres. Ações mais efetivas devem ser instituídas, buscando prevenção, diagnóstico precoce, terapêutica rápida e eficaz, adequada correção óptica, tratamento da ambliopia associada à estimulação visual.

\section{CONCLUSÃO}

Maiores informações sobre catarata congênita devem ser dadas aos familiares e pediatras buscando diagnóstico e tratamento precoces.

Apesar da grande evolução das técnicas cirúrgicas, o resultado funcional ainda permanece inadequado: atraso na realização da facectomia, não correção óptica pós-cirurgia e o tratamento inadequado da ambliopia, tornando o tratamento da catarata infantil um grande desafio.

\section{ABSTRACT}

Purpose: To establish the frequency of infantile cataract in the Low Vision Service of São Geraldo Hospital (HSGHCUFMG) and to analyze basis of history, ophthalmologic examination and optical prescription in these patients. Methods: Retrospective study of infantile cataract recorded in the Low Vision Service from January/1992 to December/2002. Age, sex, race, age at the first evidence of leukocoria and who noticed it, ages at diagnosis and phacectomy, family history of cataract, intraocular lens implantation, visual acuity (VA) and optic prescription were evaluated. Results: There were 44 patients. The age ranged from 0-15 years: 19 (43.0\%) 0-3, 14 (32.0\%) 4-10 and 11 (25.0\%) from 11-15 years. Twenty-eight $(63.6 \%)$ were female and 19 (43.2\%) leukodermic. The first evidence of leukocoria was observed by the mother in 17 patients $(38.6 \%)$. Leukocoria was observed in the first 2 months of life in $45.5 \%$ of the patients; phacectomy was performed in $43.2 \%$ of the patients with more than 1 year of age. Optic prescription was performed in $61.4 \%$ of the cases with $9.1 \%$ of the patients below 1 year of age. Intraocular lens was implanted in $13.6 \%$ and 4 patients $(9.1 \%)$ had a family history of congenital cataract. In $20.4 \%$ VA was lower than $20 / 400$ and secondary glaucoma was observed in $18.2 \%$ of the cases. Low vision aids included two glasses and one telescope. Conclusion: More information about congenital cataract should be provided for families and pediatricians, and early surgical treatment, optic correction and treatment of amblyopia should be performed promptly.

Keywords: Cataract/congenital; Cataract/diagnosis; Cataract/ epidemiology; Vision low

\section{REFERÊNCIAS}

1. Curso de Salud Ocular Comunitaria Pro Vision 2, 2002. In: Manual de Salud Ocular Comunitaria Pro Vision 2. Londres: Escuela de Salud Publica y Medicina Tropical; 2002. p.32-6.

2. Arieta C, José NK. Catarata congênita: dificuldades no tratamento. Arq Bras Oftalmol. 1987;50(3):116-9.

3. Murta J. Catarata pediatrica. Coimbra: Ediliber; 1998.

4. Reis PA, Campos CM, Fernandes LC. Características da população portadora de visão subnormal do Hospital São Geraldo. Um estudo retrospectivo de 435 casos. Rev Bras Oftalmol. 1998;57(4):287-94.

5. Carvalho KM, Minguini N, Moreira Filho DC, Kara-José N. Characteristics of a pediatric low-vision population. J Pediatr Ophthalmol Strabismus. 1998; $35(3): 162-5$.

6. Onuki-Haddad MA, Braga MSAP, Sampaio MW, Kara-Jose N. Causes of visual impairment in childhood and adolescence: A retrospective study of 1917 cases. In: Stuen C, Arditi A, Horowitz A, Lang MA, Bruce R, Seidman KL. Vision rehabilitation assessment, intervention and outcomes. New York: Swets \& Zeitlinger; 2000. p.371-5

7. Brito PR, Veitzman S. Causas de cegueira e baixa visão em crianças. Arq Bras Oftalmol. 2000;63(1):49-52.

8. Pereira MVC, Fernandes LC. Catarata congênita: diagnóstico e tratamento precoces [Poster]. In: Congresso Internacional de Catarata e Cirurgia Refrativa, 3, 1996; Belo Horizonte - Brasil.

9. Leal DB, Tavares SS, Ventura LO, Florêncio T. Atendimento a portadores de visão subnormal: estudo retrospectivo de 317 casos. Arq Bras Oftalmol. 1995;58(6):439-42.

10. Hyvarinen L. Vision in children normal and anormal. Ontario: The Canadian Deaf-Blind \& Rubella Association;1988. 64p.

11. Hing S, Speedwell L, Taylor D. Lens surgery in infancy and childhood. Br J Ophthalmol. 1990;74(2):73-7. Comment in: Br J Ophthalmol. 1990;74(2):66.

12. Magnusson G, Abrahamsson M, Sjostran J. Changes in visual acuity from 4 to 12 years of age in children operated for bilateral congenital cataracts. Br J Ophthalmol. 2002;86(12):1385-9.

13. Birch EE, Stager D, Leffler J, Weakley D. Early treatment of congenital 
unilateral cataract minimizes unequal competition. Invest Ophthalmol Vis Sci. 1998;39(9):1560-6.

14. Lee YC, Kim HS. Clinical symptoms and visual outcome in patients with presumed congenital cataract. J Pediatr Ophthalmol Strabismus. 2000;37(4): 219-24.

15. Yorston D, Wood M, Foster A. Results of cataract surgery in young children in east Africa. Br J Ophthalmol. 2001;85(3):267-71. Comment in: Br J Ophthalmol. 2001;85(3):254.

16. Tartarella MB, Kawakami LT, Scarpi MJ, Hayashi S, Bonomo PPO. Aspectos cirúrgicos em catarata congênita. Arq Bras Oftalmol. 1995;58(1):24-8
17. Yamamoto M, Dogru M, Nakamura M, Shirabe H, Tsukahara Y, Sekiya Y. Visual function following congenital cataract surgery. Jpn J Ophthalmol. 1998;42(5):411-6.

18. Autrata R, Rehurek J, Uncovska E, Vancurová J. Binocular vision after cataract surgery in children - long term results. Cesk Slov Oftalmol. 2001; 57(2):92-8.

19. Faye EE. Low vision child. In: Faye EE. Clinical low vision. $2^{\text {nd }}$ Ed Boston: Little, Brown; 1984. p.437-75.

20. Katz B, Sireteanu R. [The Teller Acuity Card Test: possibilities and limits of clinical use]. Klin Monatsbl Augenheilkd. 1989;195(1):17-22. 